\title{
Délos : l'Agora des Compétaliastes et ses abords
}

Étude de la vaisselle céramique et des lampes

\section{Claire Hasenohr et Anne-Sophie Martz}

\section{(2) OpenEdition}

\section{Journals}

Édition électronique

URL : https://journals.openedition.org/baefe/3889

DOI : $10.4000 /$ baefe.3889

ISSN : 2732-687X

Éditeur

ResEFE

Référence électronique

Claire Hasenohr, Anne-Sophie Martz, « Délos : l'Agora des Compétaliastes et ses abords » [notice archéologique], Bulletin archéologique des Écoles françaises à l'étranger [En ligne], Grèce, mis en ligne le 27 juillet 2021, consulté le 04 août 2021. URL : http://journals.openedition.org/baefe/3889; DOI : https://doi.org/10.4000/baefe.3889

Ce document a été généré automatiquement le 4 août 2021.

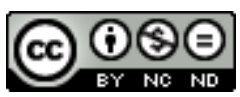

Le Bulletin archéologique des Écoles françaises à l'étranger est mise à disposition selon les termes de la Licence Creative Commons Attribution - Pas d'Utilisation Commerciale - Pas de Modification 4.0 International. 


\section{Délos : l'Agora des Compétaliastes et ses abords}

Étude de la vaisselle céramique et des lampes

Claire Hasenohr et Anne-Sophie Martz

\section{NOTE DE L'AUTEUR}

Date précise de l'opération : 2 janvier-29 mai 2020

Numéro de mission : $\mathrm{C08}$

Composition de l'équipe de terrain : La mission était une mission d'étude hors terrain, menée à domicile pendant le confinement COVID.

Établissement éditeur : EFA, Ephorie des antiquités des Cyclades

Établissements porteurs de l'opération : EFA

Données scientifiques produites : numéro de bordereau de versement aux archives : 2020004

Chroniques de l'EfA :

https://chronique.efa.gr/?kroute=report\&id=727

\section{Phasage chronologique de l'Agora des Compétaliastes et de ses abords}

1 Les travaux menés sur l'Agora des Compétaliastes et ses abords par $\mathrm{Cl}$. Hasenohr entre 1995 et $2007^{1}$ ont permis de définir un phasage des constructions. Il est apparu que les zones Est et Sud du secteur connaissent une occupation ancienne, dès l'époque géométrique et archaïque, et que l'agora est aménagée plus tardivement sur une zone de plage et de lagune d'eau douce. Une première place publique est installée au Sud du Portique de Philippe, probablement dès la fin du $\mathrm{III}^{\mathrm{e}}$ siècle ou au début $d u{ }^{\mathrm{e}}{ }^{\mathrm{e}}$ siècle 
av. J.-C. L'esplanade est agrandie et dallée dans un deuxième temps, en lien avec les travaux menés par l'épimélète Théophrastos avant 125 av. J.-C. et peut-être même au-delà Ces deux états de la place sont attestés par l'épigraphie qui évoque les importants remblais nécessaires à leur mise en œuvre. À l'époque romaine impériale, l'agora est progressivement envahie par des habitations. Un ensemble thermal est construit dans la zone sud, au plus tôt au IV ${ }^{\mathrm{e}}$ siècle ap. J.-C. Enfin, des maisons modestes sont implantées dans le secteur à l'époque protobyzantine. Même si les contextes tardifs ont été, comme ailleurs à Délos, largement éliminés par les premiers travaux de terrain au début du $\mathrm{xx}^{\mathrm{e}}$ siècle, et même si la vaisselle de la fin de l'époque hellénistique est, comme très souvent sur l'île, largement majoritaire, le matériel présenté dans ce dossier couvre, de façon plus ou moins abondante, toutes ces périodes (fig. 1). De plus, il est issu, dans la plupart des cas, de contextes stratigraphiques bien définis, antérieurs à la période hellénistique tardive, ce qui est assez rare à Délos pour être souligné.

Fig. 1. Mobilier céramique issu des fouilles de l'Agora des Compétaliastes et ses abords.

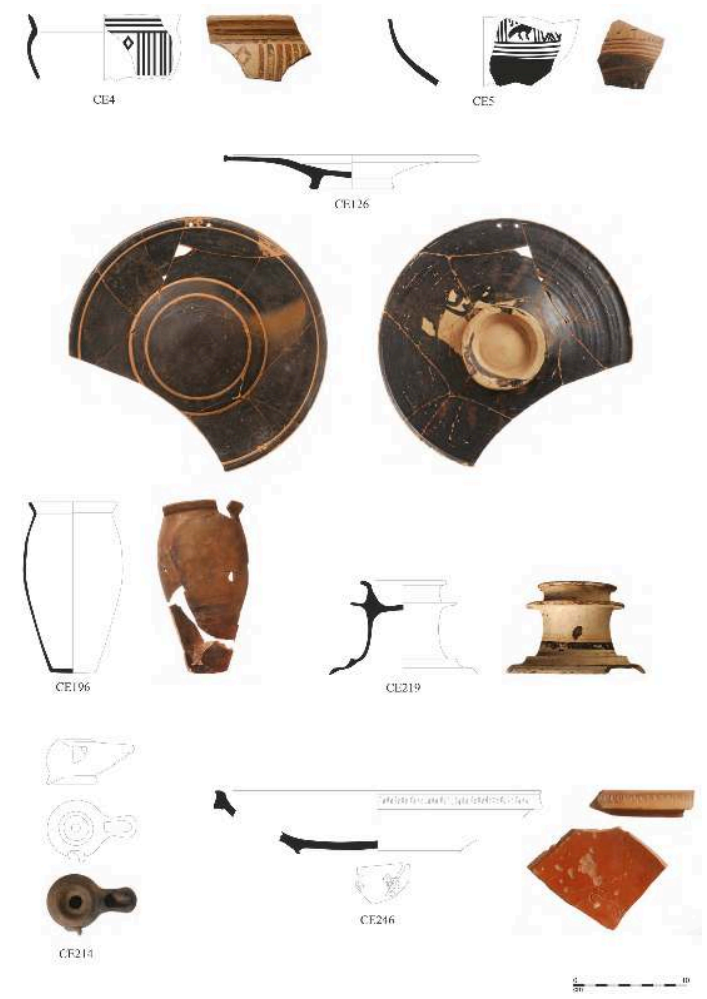

CE4 et CE5 : skyphoi, Géométrique récent I - II. CE126 : plat à décor West Slope, II s. av. J.-C. CE196 : gobelet à paroi fine, de type Ricci 1/7 ; CE219 : thymiatérion en « Lagynos ware »; CE214 : lampe tournée de type «Délos », $\|_{-1}$ err $^{\text {S }}$. av. J.-C. CE246 : plat en sigillée phocéenne, de type Hayes 3, entre le début du ve $\mathrm{s}$. et le troisième quart du vi $\mathrm{s}$. ap. J.-C.

Dessins : Chr. Mitsopoulou, Ch. Carrato, S. Gauduchon, N. Sigalas. Photographies: Ph. Collet, pour EfA. Lieu de stockage des données: Archives EFA.

\section{Objectif de l'étude}

2 L'objectif de l'étude de la vaisselle céramique issue des fouilles menées sur l'Agora des Compétaliastes et ses abords est de proposer des datations absolues ${ }^{2}$ aux assemblages 
de matériel, afin de favoriser la compréhension de la chronologie de la place et des constructions qui la bordent.

3 L'analyse typologique, économique ou fonctionnelle de ce mobilier ne faisait pas l'objet de cette première phase d'étude. Pour ces questions, nous renvoyons le lecteur aux sources bibliographiques et à l'état de l'art propre à chaque catégorie de matériel. Nous considérons toutefois que cette présentation chronologique de la vaisselle céramique issue de l'Agora des Compétaliastes constitue une nouvelle étape dans la connaissance du matériel céramique trouvé à Délos, et nous espérons que cet abondant mobilier sera étudié de façon plus complète dans les années à venir.

\section{Méthode de travail}

4 L'étude de la vaisselle céramique issue des travaux récents menés sur l'Agora des Compétaliastes et ses abords a été commencée en 1995 par Chr. Mitsopoulou : jusqu'en 2007, elle a sélectionné, inventorié, dessiné ${ }^{3}$ et analysé le matériel. A. Peignard-Giros, G. Finkelstein, F. Blondé, J.-S. Gros, A. Tichit et G. Ackermann ont aimablement proposé quelques observations complémentaires sur ce mobilier. Le dossier, qui intègre l'ensemble de ces données, remis à l'École française d'Athènes, a été finalisé par A.-S. Martz entre janvier et mai 2020.

5 Le matériel mis au jour entre 1995 et 2007 est actuellement conservé au musée de Délos, à l'exception d'une partie des tessons de vaisselle commune ou d'amphores qui a fait l'objet d'un tri sur le terrain, au moment des fouilles ${ }^{4}$. Certaines US ont livré d'énormes quantités de tessons, d'autres de très modestes assemblages, mais le matériel n'a pas fait l'objet de comptages systématiques.

6 Pour inventaire, Chr. Mitsopoulou a sélectionné prioritairement - mais pas uniquement - la vaisselle de table: ces productions de vaisselle dite «fine» sont abondamment étudiées, leur chronologie est assez bien établie, et elles se reconnaissent facilement dans les assemblages de matériel. Cette catégorie de vaisselle constitue donc un très solide point de départ à une analyse chronologique.

7 Trois avertissements préliminaires doivent être formulés. D'abord, le matériel, conservé au musée de Délos, n'a pas été vu par l'auteur de ce dossier. Le travail a consisté en une synthèse et une analyse des données brutes fournies par Chr. Mitsopoulou : notes de travail, photographies, dessins. Ensuite, le matériel étudié est, dans la grande majorité des cas, très fragmentaire, issu de stratigraphies parfois complexes, souvent perturbées par de nombreuses constructions, et son identification est difficile. Enfin, les conditions sanitaires particulières du premier semestre 2020 ont compliqué l'accès aux ressources bibliographiques nécessaires au bon déroulement d'un tel travail. Pour ces différentes raisons, nous prenons l'entière responsabilité de toute erreur d'interprétation qui apparaîtra dans ce dossier.

\section{Résultats}

8 L'analyse de l'ensemble des données disponibles nous a permis d'établir deux outils complémentaires: d'une part, un catalogue chrono-typologique, d'autre part, un tableau récapitulatif de tous les assemblages documentés. 
9 Le catalogue chrono-typologique présente les 248 objets qui nous ont semblé les plus représentatifs - dont le type est identifiable et/ou qui ont un intérêt chronotypologique particulier - parmi les objets ou tessons inventoriés par Chr. Mitsopoulou. Afin de justifier les datations proposées, nous avons toutefois indiqué, dans les tableaux de présentation des assemblages, tous les éléments à valeur chronologique. Ces objets sont classés selon les grandes périodes historiques, de l'époque géométrique à l'époque protobyzantine. Un tel classement est certes artificiel : il ne correspond ni aux réalités chronologiques des productions céramiques, ni aux phases propres à l'histoire de l'Agora des Compétaliastes. Il oblitère, aussi, la longévité de certains types. Il nous a semblé être, toutefois, la manière la plus simple de présenter un matériel qui couvre près de quinze siècles d'histoire.

Pour chaque objet, la datation est proposée en fonction de l'état de l'art et/ou de la cohérence de l'assemblage livré par l'US, avec un renvoi systématique aux sources bibliographiques consultées.

11 Si le catalogue chrono-typologique est l'outil essentiel d'une étude céramologique, le tableau récapitulatif, sondage par sondage, et US par US, de tous les assemblages de mobilier céramique documentés (fig. 2) est particulièrement utile pour la compréhension de la chronologie de la place et des constructions qui la bordent. Les informations liées à la fouille (stratigraphie, nature des US, etc.), fournies par $\mathrm{Cl}$. Hasenhor, ont servi de base à l'élaboration de ces tableaux. Dans les descriptions sommaires des assemblages sont évoqués tous les fragments présentant un intérêt chronologique, qu'ils aient été sélectionnés pour le catalogue ou non. La datation des assemblages est basée sur les informations stratigraphiques relatives à chaque sondage, sur la synthèse de la datation du matériel céramique par l'état de l'art et sur la présence/ absence de certaines productions ou types qui servent de marqueurs chronologiques ${ }^{5}$.

Fig. 2. Extrait du tableau récapitulatif des assemblages de vaisselle céramique.

\section{Sondage 1999-VII}

\begin{tabular}{|l|l|l|l|}
\hline $\mathrm{N}^{\circ}$ US & Datation de l'assemblage & Description sommaire & $\mathrm{N}^{\circ}$ de catalogue \\
\hline $1999-702$ & $\begin{array}{l}\text { Entre le dernier quart du } \\
\text { Ile s. et le Ier s. av. J.-C. }\end{array}$ & $\begin{array}{l}\text { Eléments caractéristiques des assemblages ayant pour } \\
\text { terminus 88 ou 69 (bec de lampe moulée à pâte grise, bol à } \\
\text { reliefs à engobe rouge, sigillées). }\end{array}$ & CE182; CE184 \\
\hline $1999-705$ & IIle s. av. J.-C. au moins. & $\begin{array}{l}\text { Seulement deux fonds à engobe noir en coulures datés. au plus } \\
\text { tôt du IIIe s. av. J-C. }\end{array}$ & $\begin{array}{l}\text { Aucun élément } \\
\text { sélectionné. }\end{array}$ \\
\hline $1999-708$ & $\begin{array}{l}\text { Entre le dernier quart du } \\
\text { Ile s. et le ler s. av. J.-C. }\end{array}$ & Tessons de sigillée (ESA) et de vaisselle à vernis noir. & $\begin{array}{l}\text { Aucun élément } \\
\text { sélectionné. }\end{array}$ \\
\hline $1999-709$ & $\begin{array}{l}\text { Au moins jusqu'au } \\
\text { dernier quart du Ile s. av. } \\
\text { J.-C. }\end{array}$ & $\begin{array}{l}\text { Vaisselle de table à vernis noir, fragment de vase à décor West } \\
\text { Slope, fragments de vaisselle sigillée. }\end{array}$ & CE187 \\
\hline $1999-712$ & $\begin{array}{l}\text { Antérieur au dernier } \\
\text { quart du Ile s. av. J.C. }\end{array}$ & $\begin{array}{l}\text { Matériel peu abondant : fragments de vaisselle à vernis noir } \\
\text { (dont une assiette), un pied d'amphore. }\end{array}$ & CE124 \\
\hline
\end{tabular}

Anne-Sophie Martz

\section{Conclusions}

Le dossier a été réalisé en vue de la publication du matériel dans le volume monographique, dirigé par $\mathrm{Cl}$. Hasenohr, qui sera consacré à l'Agora des Compétaliastes et ses abords ( $E A D 48$ ). Il sera complété prochainement par un réexamen, au musée de 
Délos, des 248 éléments sélectionnés et par l'analyse de quelques assemblages ${ }^{6}$ qui n'avaient pas été documentés antérieurement.

À partir des données établies dans le dossier, il est déjà possible de mener des analyses plus poussées sur ce matériel. Ainsi, un travail collectif a été mené récemment par $\mathrm{Cl}$. Hasenohr, Chr. Mitsopoulou et l'auteur sur l'occupation de nature domestique, attestée dans les boutiques au Sud de la place, dans la seconde moitié du $\mathrm{I}^{\mathrm{er}} \mathrm{s}$. av. J.-C. et/ou au début du $\mathrm{I}^{\mathrm{er}} \mathrm{s}$. ap. J.-C. Les résultats de ce travail ont été présentés en juin 2021 lors du $5^{\mathrm{e}}$ colloque IARPotHP, dans le cadre d'un panel consacré à Délos et à la problématique de l'utilisation des évènements déliens de 88 et 69 comme points de repère chronologiques en céramologie.

\section{BIBLIOGRAPHIE}

BLONDÉ 2007

Francine Blondé, Les céramiques d'usage quotidien à Thasos au IV siècle avant J.-C., Athènes, EFA, Études thasiennes 20, 2007.

HASENOHR 1996

Claire Hasenohr, « L’Agora des Compétaliastes », rapport sur la fouille menée en 1995, BCH 120.2, 1996, p. 901-911.

HASENOHR 2002

Claire Hasenohr, « L'Agora des Compétaliastes et ses abords à Délos : topographie et histoire d'un secteur occupé de l'époque archaïque aux temps byzantins », Revue des Études Anciennes 104.1, 2002, p. $85-110$

HASENOHR 2004-2005

Claire Hasenohr, « L’Agora des Compétaliastes », rapport sur les fouilles menées en 1999, 2002 et 2003, BCH 128-129.2, 2004-2005, p. 882-907.

HASENOHR 2008

Claire Hasenohr, « L’Agora des Compétaliastes », BCH 132.2, 2008, p. 817-822.

\section{NOTES}

1. Voir notamment HASENOHR 1996, 2002, 2004-2005 et 2008.

2. Rappelons toutefois ici les limites de la datation archéologique basée sur la vaisselle céramique, remarquablement exprimées par Fr. Blondé (BLONDÉ 2007) dans son étude du matériel de Thasos : elle insiste notamment sur la nécessaire prise en considération de la longévité des types. Selon elle, et nous souscrivons à son opinion, il est presque impossible, dans la connaissance actuelle du matériel, de proposer des datations qui soient plus précises qu'un quart de siècle.

3. Avec l'aide de Ch. Carrato, S. Gauduchon et N. Sigalas. 
4. Concerne les sondages 1995-I, 2002-VI, VIII et IX. Les tessons n'ont été ni comptés ni pesés.

5. Par exemple les canthares hellénistiques à vernis noir, les bols à relief ou les productions sigillées.

6. Le sondage 1999-III (US 304, 311, 312, 313, 315), l'US 1999-411, le sondage 2002-X (US $1008,1012,1016$ et 1021), l'US 2007-102.

INDEX

Thèmes : EFA

lieux https://ark.frantiq.fr/ark:/26678/pcrtaeZywr1Kbg, https://ark.frantiq.fr/ark:/26678/ pcrtWYp8PesHSI, https://ark.frantiq.fr/ark:/26678/pcrtXvsaqTQa4J

Année de l'opération : 2020

sujets https://ark.frantiq.fr/ark:/26678/pcrt1DMOWvDF4j, https://ark.frantiq.fr/ark:/26678/ pcrtM4tR8ZGTvw, https://ark.frantiq.fr/ark:/26678/pcrtlKNhumKaYq

\section{AUTEURS}

\section{CLAIRE HASENOHR}

Université Bordeaux Montaigne

ANNE-SOPHIE MARTZ

HiSoMa 